\title{
Article \\ ConvLSTM Network-Based Rainfall Nowcasting Method with Combined Reflectance and Radar-Retrieved Wind Field as Inputs
}

\author{
Wan Liu ${ }^{1,2}$, Yongqiang Wang ${ }^{1,2,3, *}$, Deyu Zhong ${ }^{4}$, Shuai Xie ${ }^{1,2}$ and Jijun Xu ${ }^{1,2}$ \\ 1 Changjiang River Scientific Research Institute of Changjiang Water Resources Commission, \\ Wuhan 430010, China; liuwan_98@163.com (W.L.); xieshuai@mail.crsri.cn (S.X.); xujj07@163.com (J.X.) \\ 2 Hubei Provincial Key Laboratory of Basin Water Resources and Ecological Environment, \\ Wuhan 430010, China \\ 3 Research Center on the Yangtze River Economic Belt Protection and Development Strategy, \\ Wuhan 430010, China \\ 4 State Key Laboratory of Hydroscience and Hydraulic Engineering, Tsinghua University, Beijing 100084, China; \\ zhongdy@tsinghua.edu.cn \\ * Correspondence: wangyq@mail.crsri.cn; Tel.: +86-15727062996
}

check for updates

Citation: Liu, W.; Wang, Y.; Zhong,

D.; Xie, S.; Xu, J. ConvLSTM

Network-Based Rainfall Nowcasting Method with Combined Reflectance and Radar-Retrieved Wind Field as Inputs. Atmosphere 2022, 13, 411.

https://doi.org/10.3390/

atmos13030411

Academic Editors: Hanbo Yang,

Songjun Han and Bing Gao

Received: 17 February 2022

Accepted: 1 March 2022

Published: 3 March 2022

Publisher's Note: MDPI stays neutral with regard to jurisdictional claims in published maps and institutional affiliations.

Copyright: (C) 2022 by the authors. Licensee MDPI, Basel, Switzerland. This article is an open access article distributed under the terms and conditions of the Creative Commons Attribution (CC BY) license (https:// creativecommons.org/licenses/by/ $4.0 /)$.

\begin{abstract}
Strong convection nowcasting has been gaining importance in regional security, economic development, and water resource management. Rainfall nowcasting with strong timeliness needs to effectively forecast the intensity of rainfall in a local region in the short term. The forecast performance of traditional methods is limited. In this paper, a rainfall nowcasting model based on the Convolutional Long Short-Term Memory (ConvLSTM) is proposed. Combined reflectance (CR) and the retrieved wind field are selected as the key factors for prediction. The model considers the influence of water vapor content, transport, and change on rainfall by measuring CR and the retrieved wind field. Forecast results are compared to different models and different input data schemes. The analysis shows that the CSI scores of this method can reach about 0.8 , which is $28 \%$ higher than that of the optical flow method. The ConvLSTM structure with spatial analysis and time memory can greatly enhance the predictive ability of the model, and the addition of wind field data also improves the forecasting performance of the model. Therefore, the idea of forecasting rainfall on the basis of water vapor content and water vapor transport is practical and effective, and the accuracy of a forecast can be improved by using ConvLSTM network to extract spatiotemporal features.
\end{abstract}

Keywords: rainfall nowcasting; deep learning; ConvLSTM; water vapor transport; radar-retrieved wind field

\section{Introduction}

Rainfall nowcasting refers to a rainfall weather forecast that predicts the rainfall in the target region within the short term $(0 \sim 6 \mathrm{~h})$, mainly targeting rainstorms caused by strong convection [1]. Severe convective rainfalls are the main cause of flooding and mudslides that usually lead to huge economic losses and casualties. Rainfall nowcasting can provide an effective basis for the early warning and prevention of flood and debris flow disasters, which is beneficial to regional security and economic development. Severe convective rainfalls are characterized by their short duration and high intensity, and rainfall nowcasting, thus, requires high-precision data to quickly forecast rainfall in a small region within a short period [2]. At present, most short-term rainfall prediction systems are based on meteorological radar data [3] with a temporal resolution of 5-6 min and a spatial resolution of $0.5-1.0 \mathrm{~km}$ [4]. The common rainfall nowcasting methods based on radar data involve the prediction of echo intensity at the next moment through radar echo extrapolation, and then converting the echo intensity value into rainfall intensity according to the reflectivity-rainfall rate (Z-R) relationship [5]. 
Radar echo extrapolation methods mainly include the Tracking of Radar Echoes by Correlation (TREC) technique [6] and the optical flow method [7,8]. However, these methods lack strong nonlinear mapping abilities, and they are unable to extract inherent features from a large amount of data [9] due to the nonlinearity and stochasticity of the dynamic weather system. In addition, the poor rainfall forecast effect is usually related to the difficulty of estimating actual rainfall using the Z-R relationship. The Z-R relationship reflects the empirical relation between radar reflectivity factor $Z$ and rain intensity $R$ [10]. However, rainfall intensity can be affected by season, region, and rainfall type, which can lead to a large deviation between rainfall values estimated using an empirical Z-R relationship and observed values [11].

Additionally, there are complex phase changes in the rainfall process, and radar echo can only represent transient air water content; therefore, it is difficult to establish a water balance relationship between radar echo and the fluxes that need time to accumulate, such as rainfall. Lavers [12] points out that the poor rainfall forecast effect is related to the uncertainty of the location of horizontal mass convergence. For different types of extreme rainstorms, water vapor transport is also very different [13], and the intensity of rainfall is consistent with the variation of boundary water vapor input and regional water vapor convergence [14]. It has been proven to be an effective and feasible method to predict mid-range extreme weather by using water vapor flux, which can characterize the process of water vapor transport [15]. Although the radar data cannot directly calculate the vapor flux, they contain the radial velocity of the radar echo, indicating the vapor transport. Radial velocity can be extrapolated to predict the location of future echoes [16], and future rainfall and area rainfall can be predicted by using reflectivity factor and wind profile data [17].

The deep learning method has been gradually applied to rainfall nowcasting due to its strong processing ability for massive amounts of incomplete and noisy data [18-20]. At present, the deep learning method is mainly used to analyze radar images and perform rainfall approach forecasting in two directions: radar echo extrapolation [21] and quantitative precipitation forecast [22,23]. Shi et al. [24] proposed a new Convolutional Long ShortTerm Memory (ConvLSTM) network for rainfall nowcasting, which could better capture spatiotemporal correlations. At the same time, Ballas [25] proposed a Convolutional Gated Recurrent Unit (ConvGRU) by combining a Convolutional Network and Gated Recurrent Unit (GRU) to learn the space-time features in videos. Subsequently, Shi et al. [26] improved the convolution structure of ConvGRU and proposed a new Trajectory GRU (TrajGRU) model with recursive connective structure learning ability. The model is more effective than ConvGRU in capturing temporal and spatial correlation. Wang et al. [27] proposed a new Predictive RNN (PredRNN) with a new core named the Spatiotemporal LSTM (ST-LSTM) unit. Subsequently, a Memory in Memory (MIM) [28] network for precipitation nowcasting was proposed. Sønderby et al. [29] introduced MetNet, which can forecast precipitation up to $8 \mathrm{~h}$ into the future at high spatial and temporal resolution. Inspired by the U-Net and SegNet series of deep learning models, Ayzel et al. [30] proposed RainNet for radar precipitation near-prediction. Pan et al. [31] proposed a novel deep learning model, FURENet, which is designed for extracting information from multiple input variables to make predictions. A GAN model proposed by $\mathrm{Hu}$ et al. [32] has proven to be effective in overcoming the limitations of blurry predictions. Ravuri et al. [33] proposed Deep Generative Models of Rain (DGMR) based on a conditional Generative Adversarial Network (GAN) model, and DGMR provided probabilistic short-term forecasts that were more location-accurate, while preserving the statistical properties of precipitation across different spatial and temporal scales.

According to the shortcomings and advantages of the above rainfall nowcasting methods, we propose a new rainfall nowcasting method based on deep learning. We selected combined reflectance (CR) and retrieved wind field as the key input factors with which to forecast rainfall at the rainfall station and used ConvLSTM to extract the temporal and spatial features of the data. CR and retrieved wind field can effectively reflect water 
vapor content and water vapor transportation. Furthermore, we compare the forecast results to different models and different input data schemes. It is shown that this model considerably improves forecast performance in comparison with the other methods.

The remaining of this article is organized as follows: In Section 2, we describe the algorithmic and technical aspects of the models. Section 3 presents the experimental data and results. Finally, the discussion and conclusion are provided in Section 4.

\section{Methods}

The new rainfall nowcasting method based on deep learning uses CR and retrieved wind field sequences to forecast future rainfall at the rainfall station, considering the physical basis and necessary conditions of rainfall formation. From the spatial viewpoint, $P$ measurements of a dynamical system at a given time over a spatial region with an $M \times N$ grid can be treated as a tensor $x \in R^{P \times M \times N}$. From the temporal viewpoint, a sequence of tensors $x_{1}, x_{2}, \ldots, x_{t}$ can be obtained by collecting measurements at fixed time intervals over time $t$. Thus, this rainfall nowcasting problem can be illustrated as:

$$
\hat{y}_{t}=\underset{y_{t}}{\operatorname{argmax}} p\left(y_{t} \mid x_{t-K+1}, \ldots, x_{t}\right)
$$

where $\left\{x_{t-K+1}, \ldots, x_{t}\right\}$ is the historical measurement sequence data of length $K$, and $y_{t}$ is the predicted rainfall data.

The deep learning model constructs a network based on a convolution layer and a ConvLSTM layer, which are good at learning the temporal and spatial features of data. The network learns the law of echo extrapolation and the relationship between radar echo and rainfall transformation according to a large amount of historical data. The proposed method makes full use of the ConvLSTM for the spatiotemporal data learning ability, and has a clear physical basis, with CR used to consider the effects of air moisture content on the rainfall, and the wind field to consider the influence of water vapor transportation and the change of rainfall.

\subsection{ConvLSTM}

ConvLSTM was proposed by Shi et al. [24] on the basis of a Fully Connected LSTM (FC-LSTM). FC-LSTM has the powerful function of processing time series, but when processing spatiotemporal data, it expands the data into vectors and uses fully connected calculations, which will increase the amount of network calculation and lose the spatial characteristics of data. The convolutional neural network model [34] considers the local correlation of spatial data through convolution calculations, and greatly reduces network parameters by employing parameter sharing and sparse connections. ConvLSTM combines the Convolutional Neural Network (CNN) and FC-LSTM to determine the future state of a cell in the grid using its adjacent input units and past states. The state-to-state and input-to-state in the model are realized through the convolution layer, and the spatial information retained by the convolution operation and the time information retained by the LSTM are used to output the predicted value. All inputs $\left(x_{1}, x_{2}, \ldots, x_{t}\right)$, hidden state $\left(h_{1}, h_{2}, \ldots, h_{t}\right)$, unit output $\left(c_{1}, c_{2}, \ldots, c_{t}\right)$, and the three gate controls $\left(i_{t}, f_{t}, o_{t}\right)$ in ConvLSTM are all three-dimensional tensors $R^{P \times M \times N}$, where $P$ represents the number of variables or channels of the input or state, and $M$ and $N$ represent the width and height of the input or state. When $M$ and $N$ are 1, the network is FC-LSTM. The structure diagram of ConvLSTM is shown in Figure 1. The calculation formula for the memory network at time $\mathrm{t}$ of ConvLSTM is as follows:

$$
\begin{aligned}
& i_{t}=\sigma\left(W_{x i} * x_{t}+W_{h i} * h_{t-1}+W_{c i} \circ c_{t-1}+b_{i}\right) \\
& f_{t}=\sigma\left(W_{x f} * x_{t}+W_{h f} * h_{t-1}+W_{c f} \circ c_{t-1}+b_{f}\right) \\
& o_{t}=\sigma\left(W_{x o} * x_{t}+W_{h o} * h_{t-1}+W_{c o} \circ c_{t-1}+b_{o}\right) \\
& c_{t}=f_{t} \circ c_{t-1}+i_{t} \circ \tanh \left(W_{x c} * x_{t}+W_{h c} * h_{t-1}+b_{c}\right) \\
& h_{t}=o_{t} \circ \tanh \left(c_{t}\right)
\end{aligned}
$$


where $\sigma$ represents the sigmoid activation function, * represents the convolution operator, and ${ }^{\circ}$ represents the Hadamard product.

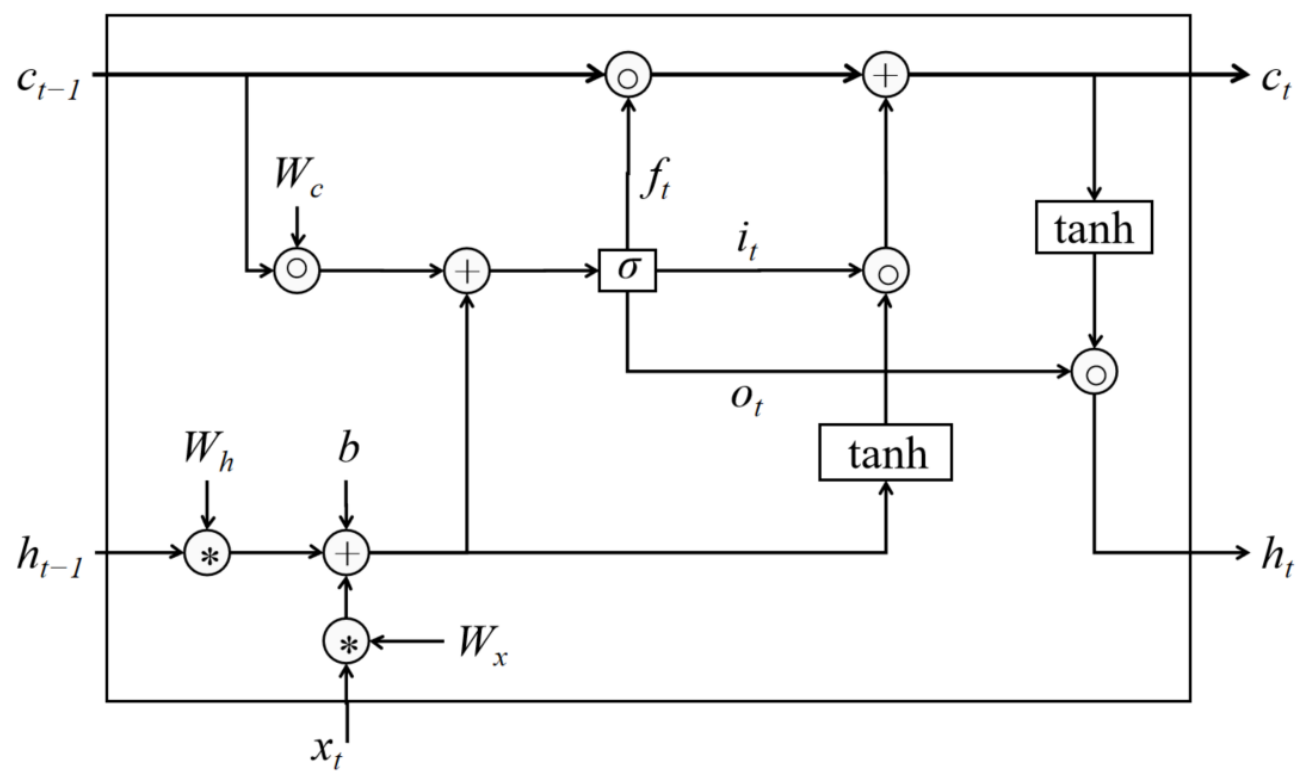

Figure 1. Schematic diagram of ConvLSTM structure. $\sigma$ and tanh are the activation function, * represents the convolution operator, ${ }^{\circ}$ represents the Hadamard product, and + represents plus.

\subsection{Input Factor Selection and Data Preprocessing}

Water vapor flux is commonly used to study water vapor transport and refers to the amount of water vapor passing through a certain section in a unit of time. Its calculation formula is as follows:

$$
Q=\frac{1}{g} \int_{p_{s}}^{0} V \cdot q d p=\frac{1}{g} \int_{p_{s}}^{0}(u, v) q d p
$$

where $Q$ is the vertical integral of horizontal water vapor flux in the air column above unit area, $V$ is the horizontal wind speed vector, $u$ and $v$ are the zonal and meridional components of wind speed, $q$ is specific humidity, $p$ is air pressure, and $p_{s}$ is surface air pressure. Furthermore, the divergence calculation of water vapor flux is carried out to obtain the divergence of water vapor flux, which is a physical quantity used to measure the convergence and divergence of water vapor in the horizontal direction. When divergence is less than 0 , it shows water vapor convergence, and strong water vapor convergence usually exists in rainstorm areas. The distribution of water vapor flux divergence has a spatial correlation with rainfall distribution. In the existing methods, water vapor flux cannot be calculated directly and easily from radar data, but there are reflectivity factors that reflect water vapor content and the radial velocity of radar echo that can invert wind field. Water vapor and its transport can be approximated based on reflectivity and radial velocity. The main flow and a schematic illustration of the model architecture are shown in Figure 2.

$\mathrm{CR}$ is selected to reflect water vapor content. In the mountainous environment, combined reflectance is preferred over other products commonly used for quantitative rainfall estimation [35], such as CAPPI, etc. The combined reflectivity factor is used to divide the echo data of an individual into several vertical columns and take the maximum echo intensity value in each column. Combined reflectance products can be provided by weather radar systems, and combined reflectance can also be calculated using meteorological analysis tools. 


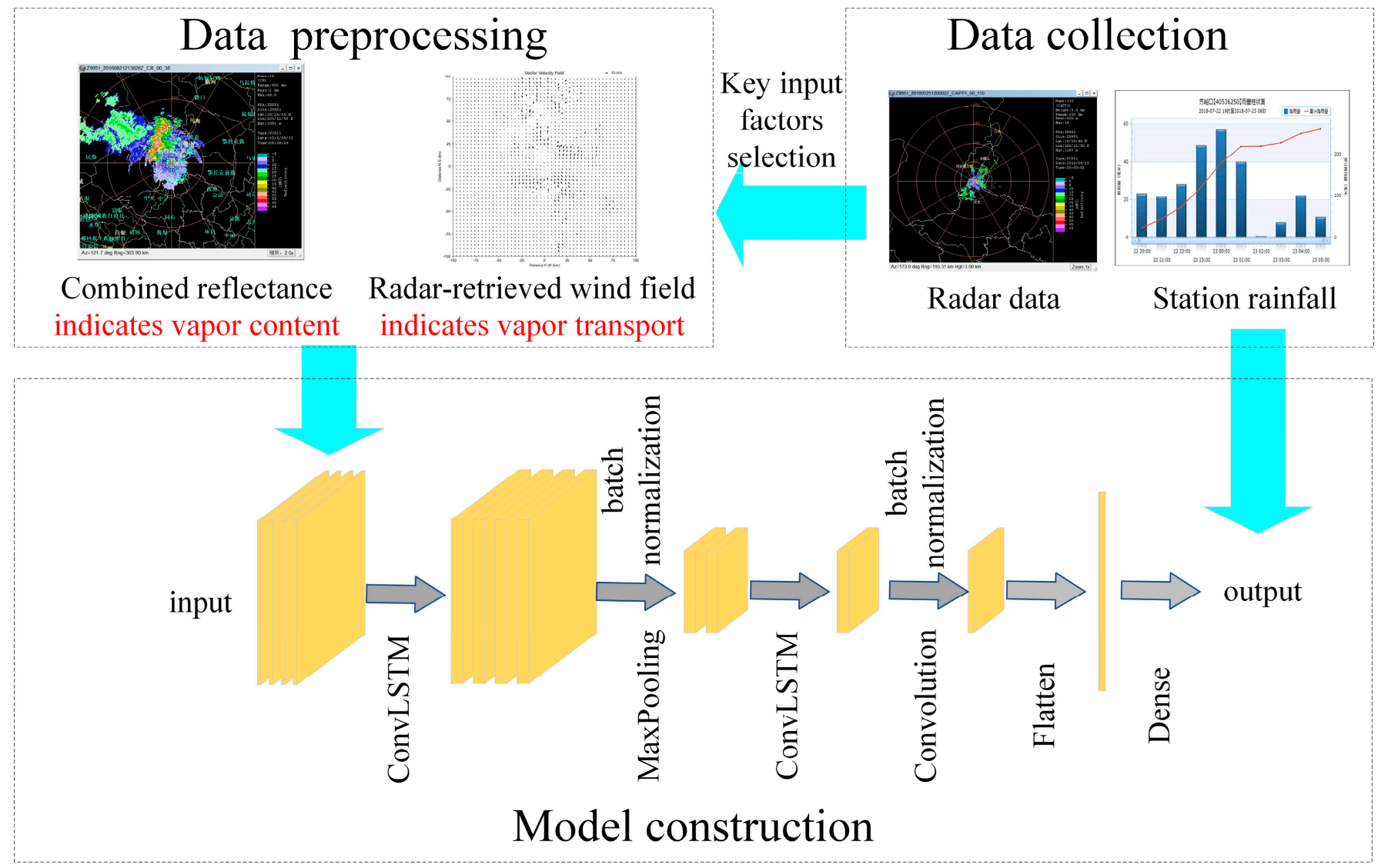

Figure 2. The main flow and a schematic illustration of the model architecture.

The two-dimensional wind field data products need to carry out the wind field inversion calculation of radar data. In this paper, the background error covariance function method [36] was used to calculate the 2D wind field. The self-covariance and crosscovariance functions were used together with statistical interpolation techniques to conduct vector wind analysis based on Doppler radial velocity observations on conical surfaces scanned by low-elevation radar. The calculated results are superior to the traditional Velocity-Azimuth Display (VAD) technique. The optimal estimate of the true vector wind field is given by the minimizer of the following cost function:

$$
J=\Delta \mathbf{a}^{\mathrm{T}} \mathbf{B}^{-1} \Delta \mathbf{a}+\sigma_{\mathrm{ob}}^{-2}(\mathbf{H} \Delta \mathbf{a}+H(\mathbf{b})-\mathbf{y})^{\mathrm{T}}(\mathbf{H} \Delta \mathbf{a}+H(\mathbf{b})-\mathbf{y})
$$

where $\mathbf{B}$ is the background error covariance matrix, $\Delta \mathbf{a}=\mathbf{a}-\mathbf{b}$ is the state vector for the analyzed incremental vector wind field, $\mathbf{a}$ and $\mathbf{b}$ are the analyzed velocities and the background velocities, respectively, $\sigma_{\mathrm{ob}}{ }^{-2}$ is the observation error variance, $\mathbf{y}$ is the state vector of the radial velocity observations, $H$ is the observation operator that maps state vectors from the analysis space to the observation space, and $\mathbf{H}$ is the linearization of $H$. The minimizer of $J$ can be obtained by solving the linear system of algebraic equations derived from $\partial J / \partial \Delta \mathbf{a}=0$.

After the combined reflectivity and two-dimensional wind fields are obtained, the data are interpolated onto a uniform grid within a specified range. Finally, the two kinds of data are spliced and sampled in chronological order to obtain the input sample $\left\{x_{t-K+1}, \ldots, x_{t-1}, x_{t}\right\}$ at time $t$. Among them, $x_{t-1}$ and $x_{t}$ are a group of combined reflectivity and wind field data with a time interval of $6 \mathrm{~min}$, a resolution of $500 \mathrm{~m} \times 500 \mathrm{~m}$, and a size of $(M, N, 3)$ for the tensor. The sample input at time $t$ corresponds to the sample output $y_{t}$, which is the rainfall measured at the rainfall station in time T after time $t$. 


\section{Results}

\subsection{Research Region Overview and Data Set}

Take Suyukou Gully at the eastern foot of Helan Mountain as an example. This area is a high-value area of rainfall and flow, with $70 \%$ annual rainfall concentrated from June to September and average annual rainfall of $200 \sim 400 \mathrm{~mm}$, which is a high-risk area for mountain flood disasters in Ningxia. The basin area is $50.5 \mathrm{~km}^{2}$ and the gully ratio drop is $73.9 \%$. The forecasting of rainstorms is of great significance to the forecast and early warning of mountain floods. There are four rainfall stations in the basin. The general situation of the basin and the distribution of stations are shown in Figure 3 . There is a great difference in rainfall among the stations, and the rainfall is mainly concentrated in the Suyukou station. Much of the time, only Suyukou has heavy rainfall, while the other three stations have little or no rainfall, so it is difficult to judge the rainstorm center.

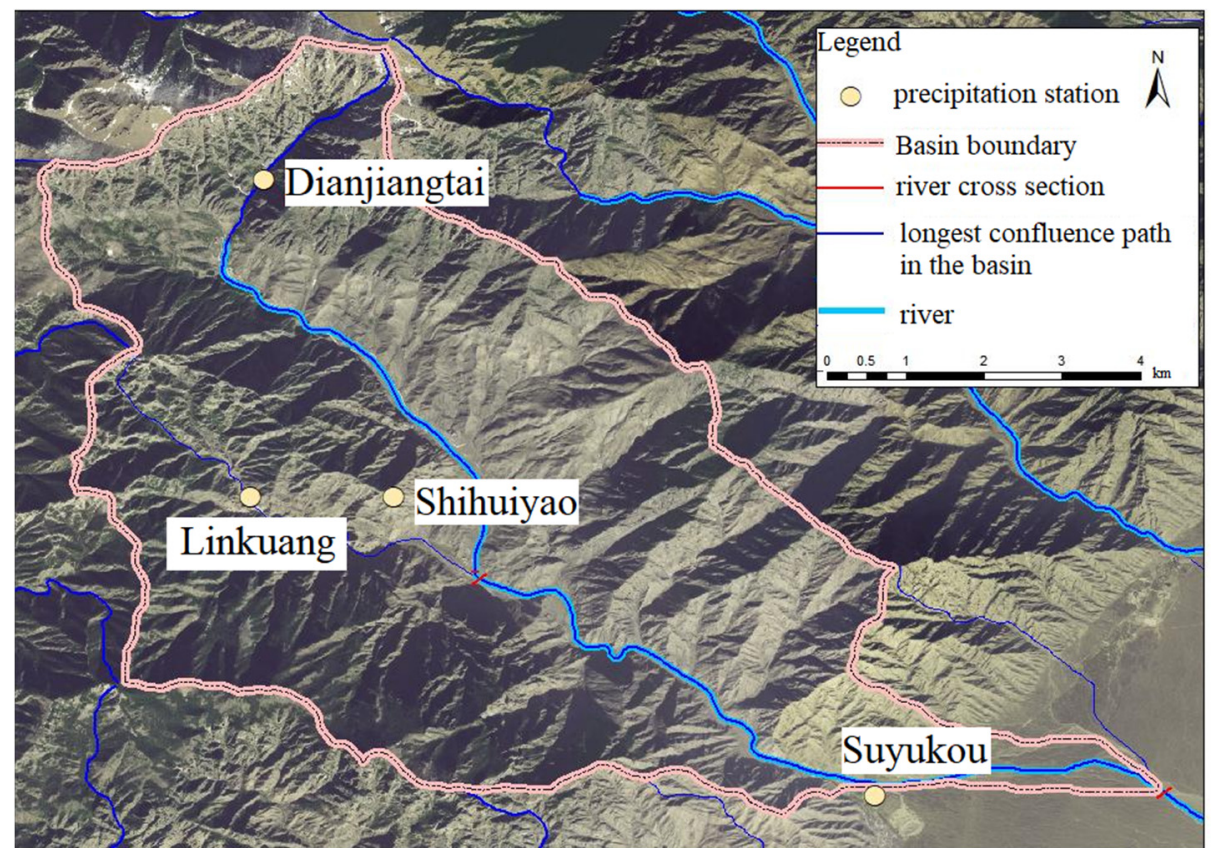

Figure 3. General situation of the basin and the distribution of rainfall stations.

In this paper, rainfall data from the rainfall stations are mainly from the Ningxia Hydrology Bureau, including data from 2015 to 2020. The radar data are obtained from the C-band dual-polarization radar of the Yinchuan Meteorological Bureau. The time interval was $6 \mathrm{~min}$, the spatial resolution was $0.5 \mathrm{~km}$, and the scanning range was $460 \mathrm{~km}$. This includes the radar base data and product data of Yinchuan station from June to September from 2017 to 2020, and some rainstorm periods from 2010 to 2018 . We selected 46 historical rainfall events from 2015 to 2020 to form our data set. The prediction factors are cropped to a $40 \times 30$ grid covering the target region in intervals of approximately $6 \mathrm{~min}$. A four-framewide stride-one sliding window is applied to generate 3606 sequence inputs. The sample output $y_{t}$ is the rainfall measured at the rainfall station in the future T hours. Finally, $80 \%$ of each rainfall was selected as training set samples, and the rest were test set samples.

\subsection{Setup of the Experiment}

We established the following six model schemes to evaluate the performance of our method with respect to the radar echo data set. The model scheme settings are shown in Table 1. 
Table 1. The model scheme settings.

\begin{tabular}{ccc}
\hline Model Scheme & Model Structure & Model Inputs \\
\hline Scheme 1 & ConvLSTM $^{1}$ model & $\mathrm{CR}^{2}$, radar-retrieved wind field \\
Scheme 2 & Convolution model & $\mathrm{CR}$, radar-retrieved wind field \\
Scheme 3 & LSTM $^{3}$ model & $\mathrm{CR}$, radar-retrieved wind field \\
Scheme 4 & ConvLSTM model & CR \\
Scheme 5 & ConvLSTM model & CR, radar radial velocity \\
Scheme 6 & \multicolumn{2}{c}{ Optical flow method } \\
\hline
\end{tabular}

${ }^{1}$ Convolutional Long Short-Term Memory (ConvLSTM). ${ }^{2}$ Combined Reflectance (CR). ${ }^{3}$ Long Short-Term Memory (LSTM). ${ }^{4}$ Optical flow method computes the optical flow of the last two observed frames and performs semi-Lagrangian advection afterwards. Finally, the Z-R relationship is used to estimate the rainfall at the grid point where the station is located.

The ConvLSTM model uses the ConvLSTM network to learn the temporal and spatial features of the data, and a convolutional network to decode the data. The network structure parameter information of the ConvLSTM model is shown in Table 2. The last two layers are fixed as a Flatten layer and a full connection layer. The Flatten layer is used to reduce the dimension of the output of the convolution layer to facilitate the calculation of the full connection layer. At the same time, a batch normalization layer (BN) was added after each convolutional layer and ConvLSTM layer to speed up the training process and improve performance. The convolution model can be obtained by replacing the ConvLSTM network with a Conv3D network, with a convolution kernel of $(3,3,3)$. The LSTM model first compresses the spatial information of the data to one dimension. It includes a three-layer LSTM containing 32 hidden states and a full connection layer.

Table 2. Network structure parameter information table of the ConvLSTM model.

\begin{tabular}{cccc}
\hline Network Layer Type & Kernel Size & Network Layer Output Size & Network Layer Parameters \\
\hline conv_lstm2d (ConvLSTM2D) & $(5,5)$ & (None, $4, M, N, 16)$ & 30,464 \\
batch_normalization (BN) & Null & (None, $4, M, N, 16)$ & 64 \\
max_pooling3d (MaxPooling3D) & Null & (None, $2, M / 2, N / 2,16)$ & 0 \\
conv_lstm2d_1 (ConvLSTM2D) & $(5,5)$ & (None, $M / 2, N / 2,16)$ & 51,264 \\
batch_normalization_1 (BN) & Null & (None, $M / 2, N / 2,16)$ & 64 \\
conv2d (Conv2D) & $(3,3)$ & (None, $M / 2, N / 2,4)$ & 580 \\
batch_normalization_2 (BN) & Null & (None, $M / 2, N / 2,4)$ & 16 \\
flatten (Flatten) & Null & (None, $M * N)$ & 0 \\
dense (Dense) & Null & (None, 1$)$ & $M * N+1$ \\
\hline
\end{tabular}

The mean-square error (MSE) function was selected as the loss function, and the back propagation algorithm was used to calculate the error. The Adam optimization algorithm [37] optimized the loss function of the model. The batch size was set to 16, and the optimization iterations was set to 50 epochs. The mean-square error (MSE) function formula is as follows:

$$
M S E=\frac{1}{n} \sum_{i=1}^{n}\left(y_{i}-y_{i}^{\prime}\right)^{2}
$$

where, $y_{i}$ is the measured value, $y_{i}{ }^{\prime}$ is the predicted value of the model, and $n$ is the number of samples.

\subsection{Evaluation Methods}

In this paper, we use the root-mean-square error (RMSE) and correlation coefficient (CC) to evaluate the simulation accuracy of the model. The calculation formula of RMSE and correlation coefficient $C C$ are as follows:

$$
R M S E=\sqrt{\frac{1}{n} \sum_{i=1}^{n}\left(y_{i}-y_{i}^{\prime}\right)^{2}}
$$




$$
C C=\frac{\sum_{i=1}^{n}\left(y_{i}-\bar{y}\right)\left(y_{i}^{\prime}-\overline{y^{\prime}}\right)}{\sqrt{\sum_{i=1}^{n}\left(y_{i}-\bar{y}\right)^{2} \sum_{i=1}^{n}\left(y_{i}^{\prime}-\overline{y^{\prime}}\right)^{2}}}
$$

where $y_{i}$ and $y_{i}{ }^{\prime}$ are the measured value and model predicted value, respectively, $\bar{y}$ and $\overline{y^{\prime}}$ are the measured average value and model predicted average value, respectively, and $n$ is the number of samples. The value range of the correlation coefficient is $[-1,1]$. When $C C>0$, it indicates that the two variables are positively correlated; when $C C<0$, it shows a negative correlation. The larger the $\mathrm{CC}$ value is, the higher the positive correlation between $y$ and $y^{\prime}$ is, and the better the forecasting performance is.

At the same time, to analyze the effect of the model on a rainstorm forecast, several commonly used evaluation indexes of rainfall nowcasting, such as critical success index $(C S I)$, false alarm rate $(F A R)$, and probability of detection $(P O D)$, were selected to evaluate the effect of the model. CSI defines the rate at which a forecast event is accurately predicted. Firstly, a rainfall threshold $k$ needs to be set, and the samples are classified according to the relationship among measured rainfall, predicted rainfall, and threshold, as shown in Table 3. Finally, the number of successful predictions $(A)$, the number of empty predictions $(B)$, and the number of missed predictions $(C)$ are obtained. The calculation formula of CSI, $F A R$, and $P O D$ is as follows:

$$
\begin{gathered}
C S I=\frac{A}{A+B+C} \\
F A R=\frac{B}{A+B} \\
P O D=\frac{A}{A+C}
\end{gathered}
$$

Table 3. Test index classification table of rainfall nowcasting.

\begin{tabular}{ccc}
\hline \multirow{2}{*}{$\begin{array}{c}\text { Measured } \\
\text { Value }\end{array}$} & \multicolumn{2}{c}{ Predicted Value } \\
\cline { 2 - 3 }$\geq k$ & $A$ (successful prediction) & $C$ (missed prediction) \\
$<k$ & $B$ (empty prediction) & $D$ (invalid data) \\
\hline
\end{tabular}

\subsection{Result}

The root-means-square error (RMSE) and correlation coefficient (CC) results of the $1 \mathrm{~h}$-predicted results of the test set in each scheme are shown in Table 4. Dianjiangtai, Shihuiyao, Linkuang, and Suyukou are the four rainfall stations in the basin. The results show that Scheme 1 is superior to the other schemes, and the RMSE and CC of Scheme 2 and Scheme 3 are worse than that of Scheme 4 and Scheme 5. Scheme 6 performs only slightly better than Scheme 3 . In the table, the forecasting performance of different rainfall stations varies greatly. The same scheme has different applicability to different regions. The correlation coefficient of Suyukou station with the best performance is 0.965 , and that of Linkuang station is 0.837 , which is the lowest.

Taking Suyukou station as an example, hourly rainfall at time $\mathrm{T}$ in the future was obtained by subtracting the rainfall in the future $\mathrm{T}-1 \mathrm{~h}$ from the predicted rainfall in the future T hours. Figure 4 shows the correlation coefficient (CC) results of the hourly rainfall predictions. We can find that the performance of Scheme 1 is always the best for hourly rainfall predictions, followed by Scheme 5 . The correlation coefficient of Scheme 2 deteriorates rapidly as the forecast period increases. The performances of Scheme 3 and Scheme 6 are the worst, where the correlation coefficient in the first hour has the biggest gap with Scheme 1. 
Table 4. Root-mean-square error (RMSE) and correlation coefficient (CC) of different schemes.

\begin{tabular}{|c|c|c|c|c|c|c|c|c|}
\hline \multirow[b]{2}{*}{ Model } & \multicolumn{2}{|c|}{ Dianjiangtai } & \multicolumn{2}{|c|}{ Shihuiyao } & \multicolumn{2}{|c|}{ Linkuang } & \multicolumn{2}{|c|}{ Suyukou $^{1}$} \\
\hline & $\begin{array}{c}\text { RMSE } \\
(\mathrm{mm})\end{array}$ & $C C$ & $\begin{array}{c}\text { RMSE } \\
(\mathrm{mm})\end{array}$ & $C C$ & $\begin{array}{c}\text { RMSE } \\
(\mathrm{mm})\end{array}$ & $C C$ & $\begin{array}{c}\text { RMSE } \\
(\mathrm{mm})\end{array}$ & $C C$ \\
\hline Scheme 1 & 1.62 & 0.848 & 0.82 & 0.842 & 0.78 & 0.837 & 1.58 & 0.965 \\
\hline Scheme 2 & 2.32 & 0.651 & 1.57 & 0.621 & 1.19 & 0.602 & 2.29 & 0.917 \\
\hline Scheme 3 & 2.79 & 0.648 & 2.42 & 0.533 & 1.33 & 0.529 & 3.89 & 0.804 \\
\hline Scheme 4 & 2.17 & 0.703 & 1.10 & 0.676 & 1.02 & 0.698 & 2.36 & 0.911 \\
\hline Scheme 5 & 1.78 & 0.840 & 0.95 & 0.805 & 0.88 & 0.807 & 2.03 & 0.932 \\
\hline Scheme 6 & 2.26 & 0.662 & 1.91 & 0.582 & 1.27 & 0.563 & 3.04 & 0.845 \\
\hline
\end{tabular}

${ }^{1}$ Dianjiangtai, Shihuiyao, Linkuang, and Suyukou are the four rainfall stations in the basin.

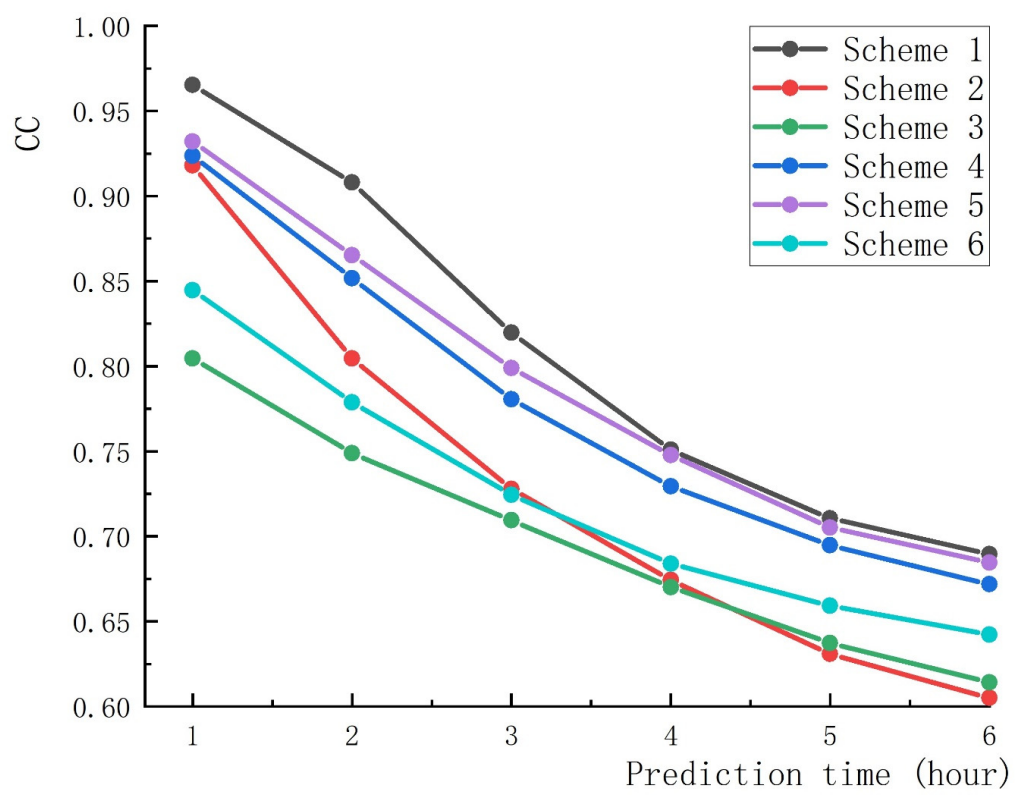

Figure 4. The correlation coefficient (CC) of the hourly rainfall forecast in Suyukou station.

When the rainfall threshold was $10 \mathrm{~mm}$ and $15 \mathrm{~mm}, C S I, F A R$, and POD of hourly rainfall prediction were calculated, as shown in Figures 5 and 6, respectively. It can be seen from the figures that all indicators change little when $k=10 \mathrm{~mm}$ and $k=15 \mathrm{~mm}$ in the same scheme. It can be found that with the increase in the length of the prediction period, the performance of each index decreases, and the change is the greatest when the prediction period is $3 \mathrm{~h}$. When the rainfall threshold is $10 \mathrm{~mm}$, the CSI of Scheme 1 decreases from 0.75 to 0.47 , and $F A R$ rises from 0.09 to 0.42 . Although the variation trend of $P O D$ was not as obvious as that of CSI and FAR, it also showed a general downward trend. This means that too long a forecast period will increase the uncertainty of nowcasting. 


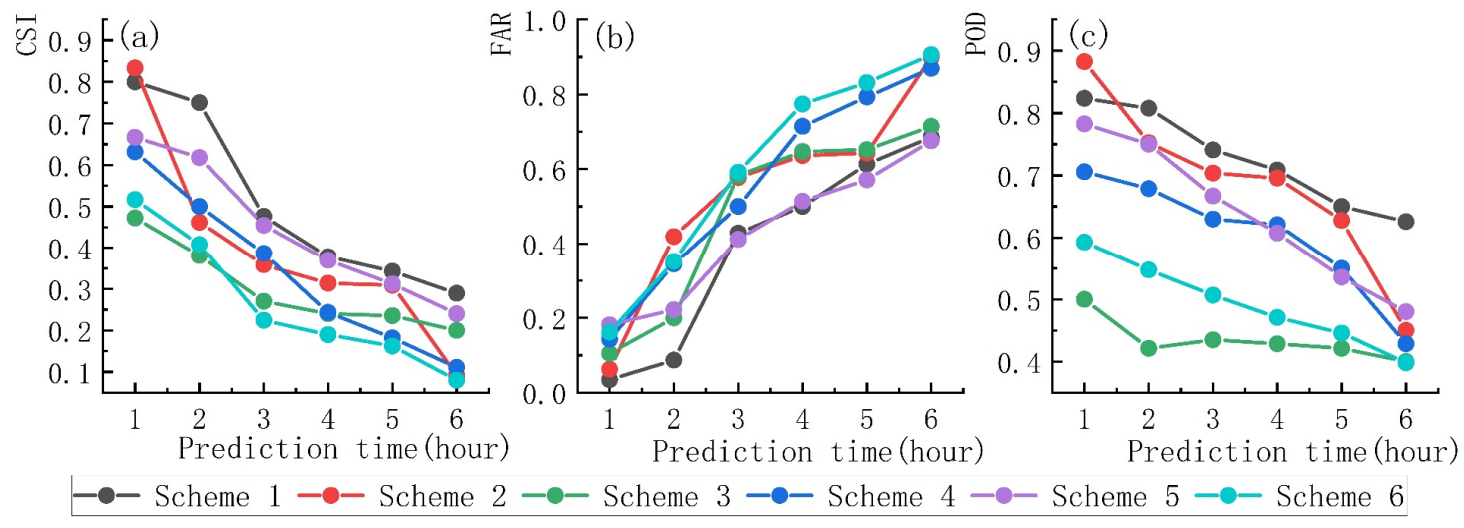

Figure 5. (a) CSI, (b) FAR, (c) POD of hourly rainfall forecast in Suyukou station when $k=10$.

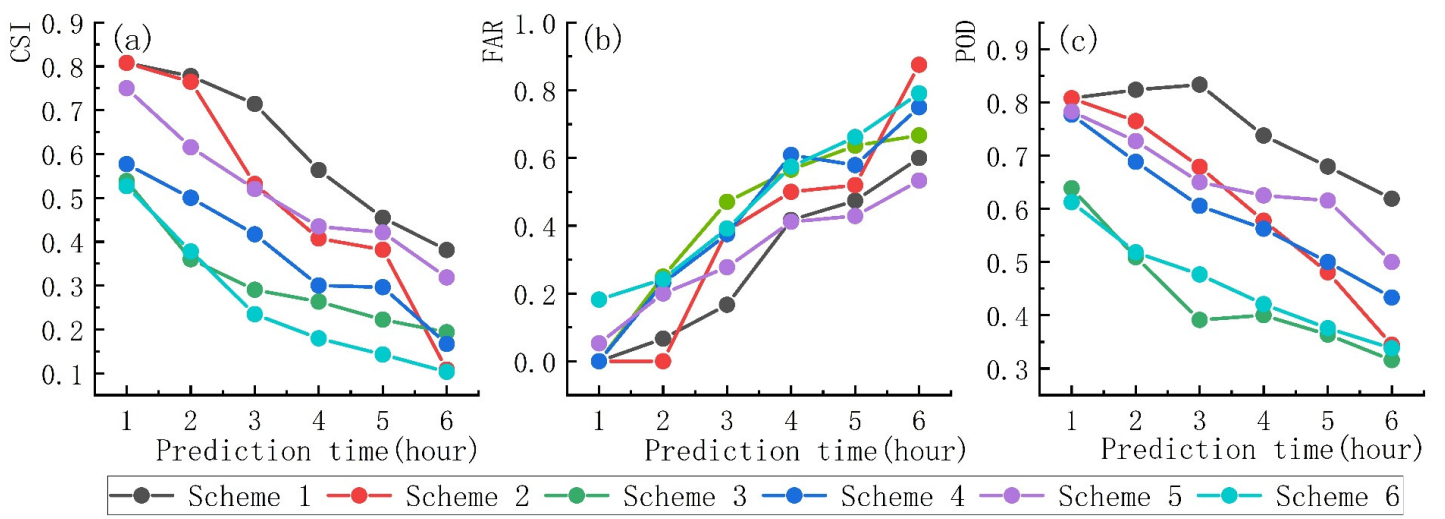

Figure 6. (a) CSI, (b) FAR, (c) POD of hourly rainfall forecast in Suyukou station when $k=15$.

\subsubsection{Model Comparison}

We compared the ConvLSTM model with the optical flow method and selected the convolution model and LSTM model as a comparative experiment against which to test the influence of the model on the forecast results. All the evaluation indexes of the ConvLSTM model, including RMSE, CC, CSI, FAR, and POD, are significantly better than the other two models. As Table 4 shows, the CC of ConvLSTM model in Suyukou station is increased by $12 \%$ compared with the optical flow method. In the other three rain stations, the RMSE and $C C$ of the models were improved more obviously than those of the other methods. As Figure 5 shows, the ConvLSTM model generally has higher CSI and POD scores and lower FAR scores than the other two models. The convolution model outperforms ConvLSTM only at $1 \mathrm{~h}$, while the LSTM model consistently performed the worst.

Figure 4 shows that Scheme 2 and Scheme 3 are worse than Scheme 4 and Scheme 5. This indicates that model adjustment has a greater impact on prediction than input adjustment. The performance of all models deteriorates as the forecast period increases, especially the convolution model, because the convolution model could not calculate time correlation and could not accurately predict the specific time of rainfall occurrence. The ConvLSTM model combines the advantages of spatial correlation captured by the convolution model and time correlation extracted by the LSTM model, which can give a better forecast of short-term precipitation. To give full play to the warning and decision-making function of the forecast results of short-term precipitation, the prediction period should not exceed $3 \mathrm{~h}$ for the case study.

\subsubsection{Comparison of Input Data}

In this work, to test the influence of input on prediction results, we selected three groups of different input predictive factor schemes, namely, Scheme 1: CR and retrieved 
wind field input, Scheme 4: single CR input, and Scheme 5: CR and radial velocity input. The results indicate that using water vapor content and water vapor transport information can better improve the rainfall nowcasting effect. As Table 4 shows, the results of Scheme 1 and Scheme 5 are better than those of Scheme 4, which indicates that the effect of adding velocity into the input, whether with radar-retrieved wind field or radial velocity, is better than that of a single CR input because velocity roughly reflects the movement of the atmosphere, which is the necessary dynamic condition for precipitation. As can be seen from Figure 4, because the same network model is used, the correlation coefficients of different input schemes have roughly the same trend with the prediction period.

As Figures 5 and 6 show, three evaluation indexes of Scheme 1 are superior to the other two schemes, especially the $P O D$ scores, which always vary widely from the other two. With the increase in the length of the prediction period, the CSI and FAR scores of Scheme 4 and Scheme 5 gradually approach those of Scheme 1 . When the prediction period is larger than $3 \mathrm{~h}$, the CSI and FAR scores of Scheme 1 and Scheme 5 are similarly poor. This illustrates that the missed times of Scheme 1 are far fewer than the successful times and empty times of prediction. When the prediction period is more than $3 \mathrm{~h}$, the performance of each index is low. Therefore, the input combination of CR and retrieved wind field is the optimal input factor combination under the condition that the forecasting performance is guaranteed.

\section{Discussion and Conclusions}

In this paper, a new deep learning-based method for rainfall nowcasting is proposed. In this method, the network is constructed based on a convolution layer and a ConvLSTM layer, which are good at learning the temporal and spatial features of data. The influence of water vapor content on rainfall is considered in relation to CR factors, and the influence of water vapor transport and change is considered on rainfall in relation to the wind field. The extrapolation law and the transforming relationship between radar echo and rainfall are learned from lots of historical data.

We compared our model with different network models and different input data combinations. The results show that the CSI scores of this method can reach about 0.8 , which is $28 \%$ higher than that of the optical flow method and the correlation coefficient is above 0.8 at each rainfall station. It is observed that the ConvLSTM structure with spatial analysis and time memory ability in the model can greatly enhance the prediction ability of the model. The addition of wind field data can also improve the forecasting performance of the model. The idea of forecasting rainfall using water vapor content and water vapor transport is practical and effective. It achieves quantitative rainfall nowcasting accurately and effectively, improving the hit rate of rainfall forecast and reducing the false alarm rate. In addition, the model has flexible data output ability and strong coupling ability with hydrological models, which has practical guiding significance for flood forecasting and dispatching in small watershed in mountainous areas.

Due to the limited data set used in this study, it is difficult to test the generalization ability of the model. In future work, we will attempt to apply it to larger data sets to test the model's practical application capabilities. Quantitative rainfall forecasting is one of the most important tasks of nowcasting. The introduction of deep learning, especially ConvLSTM, brings a new direction to the research of short-term rainfall forecast. How to use deep learning to solve the problem of segmental rainfall forecast will be the focus of future work. The inputs of this model do not consider the influence of terrain, temperature, and other factors on rainfall, so the forecasting performance of this model for different rainfall stations is quite different. In the next research work, variables such as terrain and temperature can also be considered to further study rainfall prediction. 


\begin{abstract}
Author Contributions: Conceptualization, D.Z. and Y.W.; Data curation, W.L.; Formal analysis, W.L.; Funding acquisition, Y.W. and J.X.; Investigation, W.L. and S.X.; Methodology, S.X.; Project administration, Y.W.; Resources, Y.W. and D.Z.; Software, W.L. and S.X.; Supervision, Y.W. and D.Z.; Visualization, W.L.; Writing-original draft, W.L.; Writing-review \& editing, Y.W. and J.X. All authors have read and agreed to the published version of the manuscript.
\end{abstract}

Funding: This research was funded by the Key Research and Development Program of Ningxia grant number 2020BCF01002, the National Natural Science Foundation of China grant number 51779013 and U2040212, the Fundamental Research Funds for Central Public Welfare Research Institutes grant number CKSF2021486/SZ and CKSF2019478/SZ, the National Public Research Institutes for Basic R\&D Operating Expenses Special Project grant number CKSF2017061/SZ, the Major Scientific Instrument Development Project of The National Natural Science Foundation of China grant number 51527809, and the Scientific Research Project of China Three Gorges Corporation grant number 0704106.

Institutional Review Board Statement: Not applicable.

Informed Consent Statement: Not applicable.

Data Availability Statement: Not applicable.

Conflicts of Interest: The authors declare no conflict of interest.

\title{
References
}

1. Yin, J.; Gao, Z.; Han, W. Application of a Radar Echo Extrapolation-Based Deep Learning Method in Strong Convection Nowcasting. Earth Space Sci. 2021, 8, e2020EA001621. [CrossRef]

2. Heuvelink, D.; Berenguer, M.; Brauer, C.C.; Uijlenhoet, R. Hydrological application of radar rainfall nowcasting in the Netherlands. Environ. Int. 2020, 136, 105431. [CrossRef] [PubMed]

3. Han, L.; Sun, J.; Zhang, W.; Xiu, Y.; Feng, H.; Lin, Y. A machine learning nowcasting method based on real-time reanalysis data. J. Geophys. Res. Atmos. 2017, 122, 4038-4051. [CrossRef]

4. Zhao, K.; Huang, H.; Wang, M.; Lee, W.-C.; Chen, G.; Wen, L.; Wen, J.; Zhang, G.; Xue, M.; Yang, Z.; et al. Recent Progress in Dual-Polarization Radar Research and Applications in China. Adv. Atmos. Sci. 2019, 36, 961-974. [CrossRef]

5. Alfieri, L.; Claps, P.; Laio, F. Time-dependent Z-R relationships for estimating rainfall fields from radar measurements. Nat. Hazards Earth Syst. Sci. 2010, 10, 149-158. [CrossRef]

6. Wang, G.; Wong, W.; Liu, L.; Wang, H. Application of multi-scale tracking radar echoes scheme in quantitative precipitation nowcasting. Adv. Atmos. Sci. 2013, 30, 448-460. [CrossRef]

7. Ayzel, G.; Heistermann, M.; Winterrath, T. Optical flow models as an open benchmark for radar-based precipitation nowcasting (rainymotion v0.1). Geosci. Model Dev. 2019, 12, 1387-1402. [CrossRef]

8. Woo, W.-C.; Wong, W.-K. Operational Application of Optical Flow Techniques to Radar-Based Rainfall Nowcasting. Atmosphere 2017, 8, 48. [CrossRef]

9. Chen, L.; Cao, Y.; Ma, L.; Zhang, J. A Deep Learning-Based Methodology for Precipitation Nowcasting with Radar. Earth Space Sci. 2020, 7, e2019EA000812. [CrossRef]

10. Wang, G.; Liu, L.; Ding, Y. Improvement of radar quantitative precipitation estimation based on real-time adjustments to Z-R relationships and inverse distance weighting correction schemes. Adv. Atmos. Sci. 2012, 29, 575-584. [CrossRef]

11. Shao, Y.; Fu, A.; Zhao, J.; Xu, J.; Wu, J. Improving quantitative precipitation estimates by radar-rain gauge merging and an integration algorithm in the Yishu River catchment, China. Theor. Appl. Climatol. 2021, 144, 611-623. [CrossRef]

12. Lavers, D.A.; Pappenberger, F.; Zsoter, E. Extending medium-range predictability of extreme hydrological events in Europe. Nat. Commun. 2014, 5, 5382. [CrossRef] [PubMed]

13. Yang, H.; Xu, G.-Y.; Wang, X.; Cui, C.; Wang, J.; He, D. Quantitative Analysis of Water Vapor Transport during Mei-Yu Front Rainstorm Period over the Tibetan Plateau and Yangtze-Huai River Basin. Adv. Meteorol. 2019, 2019, 6029027. [CrossRef]

14. Feng, L.; Zhou, T. Water vapor transport for summer precipitation over the Tibetan Plateau: Multidata set analysis. J. Geophys. Res. Atmos. 2012, 117, D20114. [CrossRef]

15. Lavers, D.A.; Waliser, D.E.; Ralph, F.M.; Dettinger, M.D. Predictability of horizontal water vapor transport relative to precipitation: Enhancing situational awareness for forecasting western U.S. extreme precipitation and flooding. Geophys. Res. Lett. 2016, 43, 2275-2282. [CrossRef]

16. Li, N.; Wei, M.; Niu, B.; Pan, J.; Zhang, W.; Guo, W. Application of Multiple Wind Retrieval Algorithms in Nowcasting. Atmosphere 2015, 6, 834-849. [CrossRef]

17. Ramírez, M.C.V.; de Campos Velho, H.F.; Ferreira, N.J. Artificial neural network technique for rainfall forecasting applied to the São Paulo region. J. Hydrol. 2005, 301, 146-162. [CrossRef]

18. Ayzel, G.; Heistermann, M.; Sorokin, A.; Nikitin, O.; Lukyanova, O. All convolutional neural networks for radar-based precipitation nowcasting. Procedia Comput. Sci. 2019, 150, 186-192. [CrossRef] 
19. Agrawal, S.; Barrington, L.; Bromberg, C.; Burge, J.; Gazen, C.; Hickey, J. Machine Learning for Precipitation Nowcasting from Radar Images. arXiv 2019, arXiv:1912.12132. Available online: https://ui.adsabs.harvard.edu/abs/2019arXiv191212132A (accessed on 1 December 2019).

20. Reichstein, M.; Camps-Valls, G.; Stevens, B.; Jung, M.; Denzler, J.; Carvalhais, N.; Prabhat. Deep learning and process understanding for data-driven Earth system science. Nature 2019, 566, 195-204. [CrossRef] [PubMed]

21. Sokol, Z.; Kitzmiller, D.; Pesice, P.; Mejsnar, J. Comparison of precipitation nowcasting by extrapolation and statistical-advection methods. Atmos. Res. 2013, 123, 17-30. [CrossRef]

22. Ko, C.-M.; Jeong, Y.Y.; Lee, Y.-M.; Kim, B.-S. The Development of a Quantitative Precipitation Forecast Correction Technique Based on Machine Learning for Hydrological Applications. Atmosphere 2020, 11, 111. [CrossRef]

23. Huang, H.; Zhao, K.; Zhang, G.; Lin, Q.; Wen, L.; Chen, G.; Yang, Z.; Wang, M.; Hu, D. Quantitative Precipitation Estimation with Operational Polarimetric Radar Measurements in Southern China: A Differential Phase-Based Variational Approach. J. Atmos. Ocean. Technol. 2018, 35, 1253-1271. [CrossRef]

24. Shi, X.; Chen, Z.; Wang, H.; Yeung, D.-Y.; Wong, W.-K.; Woo, W.-C. Convolutional LSTM Network: A Machine Learning Approach for Precipitation Nowcasting. arXiv 2015, arXiv:1506.04214. Available online: https://ui.adsabs.harvard.edu/abs/2015arXiv150 604214S (accessed on 1 June 2015).

25. Ballas, N.; Yao, L.; Pal, C.; Courville, A. Delving Deeper into Convolutional Networks for Learning Video Representations. arXiv 2015, arXiv:1511.06432. Available online: https:/ / ui.adsabs.harvard.edu/abs/2015arXiv151106432B (accessed on 1 November 2015).

26. Shi, X.; Gao, Z.; Lausen, L.; Wang, H.; Yeung, D.-Y.; Wong, W.-K.; Woo, W.-C. Deep Learning for Precipitation Nowcasting: A Benchmark and A New Model. arXiv 2017, arXiv:1706.03458. Available online: https://ui.adsabs.harvard.edu/abs/2017arXiv1 70603458 S (accessed on 1 June 2017).

27. Wang, Y.; Long, M.; Wang, J.; Gao, Z.; Yu, P.S. PredRNN: Recurrent neural networks for predictive learning using spatiotemporal LSTMs. In Proceedings of the 31st International Conference on Neural Information Processing Systems, Long Beach, CA, USA, 4-9 December 2017; pp. 879-888.

28. Wang, Y.; Zhang, J.; Zhu, H.; Long, M.; Wang, J.; Yu, P.S. Memory in memory: A predictive neural network for learning higher-order non-stationarity from spatiotemporal dynamics. In Proceedings of the IEEE/CVF Conference on Computer Vision and Pattern Recognition, Long Beach, CA, USA, 16-20 June 2019; pp. 9154-9162.

29. Sønderby, C.K.; Espeholt, L.; Heek, J.; Dehghani, M.; Oliver, A.; Salimans, T.; Agrawal, S.; Hickey, J.; Kalchbrenner, N. MetNet: A Neural Weather Model for Precipitation Forecasting. arXiv 2020, arXiv:2003.12140. Available online: https://ui.adsabs.harvard. edu/abs/2020arXiv200312140K (accessed on 1 March 2020).

30. Ayzel, G.; Scheffer, T.; Heistermann, M. RainNet v1.0: A convolutional neural network for radar-based precipitation nowcasting. Geosci. Model Dev. 2020, 13, 2631-2644. [CrossRef]

31. Pan, X.; Lu, Y.; Zhao, K.; Huang, H.; Wang, M.; Chen, H. Improving Nowcasting of Convective Development by Incorporating Polarimetric Radar Variables into a Deep-Learning Model. Geophys. Res. Lett. 2021, 48, e2021GL095302. [CrossRef]

32. Hu, Y.; Chen, L.; Wang, Z.; Pan, X.; Li, H. Towards a More Realistic and Detailed Deep-Learning-Based Radar Echo Extrapolation Method. Remote Sens. 2021, 14, 24. [CrossRef]

33. Chirigati, F. Accurate short-term precipitation prediction. Nat. Comput. Sci. 2021, 1, 709. [CrossRef]

34. Han, L.; Sun, J.; Zhang, W. Convolutional Neural Network for Convective Storm Nowcasting Using 3-D Doppler Weather Radar Data. IEEE Trans. Geosci. Remote Sens. 2019, 58, 1487-1495. [CrossRef]

35. Franch, G.; Maggio, V.; Coviello, L.; Pendesini, M.; Jurman, G.; Furlanello, C. TAASRAD19, a high-resolution weather radar reflectivity dataset for precipitation nowcasting. Sci. Data 2020, 7, 234. [CrossRef] [PubMed]

36. Xu, Q.; Liu, S.; Xue, M. Background error covariance functions for vector wind analyses using Doppler-radar radial-velocity observations. Q. J. R. Meteorol. Soc. 2006, 132, 2887-2904. [CrossRef]

37. Kingma, D.P.; Ba, J. Adam: A method for stochastic optimization. arXiv 2014, arXiv:1412.6980. Available online: https: / / ui.adsabs.harvard.edu/abs/2014arXiv1412.6980K (accessed on 1 December 2014). 